Mots. Les langages du politique

Gilbert Rist (dir.), Les mots du pouvoir, sens et nonsens de la rhétorique internationale

Fabienne Pierre

\title{
OpenEdition
}

Journals

Édition électronique

URL : https://journals.openedition.org/mots/3613

DOI : $10.4000 /$ mots.3613

ISSN : 1960-6001

Éditeur

ENS Éditions

Édition imprimée

Date de publication : 1 juillet 2004

Pagination : 138-141

ISBN : 2-84788-057-7

ISSN : 0243-6450

Référence électronique

Fabienne Pierre, « Gilbert Rist (dir.), Les mots du pouvoir, sens et non-sens de la rhétorique internationale », Mots. Les langages du politique [En ligne], 75 | 2004, mis en ligne le 23 avril 2008, consulté le 23 avril 2022. URL : http://journals.openedition.org/mots/3613 ; DOI : https://doi.org/ $10.4000 /$ mots.3613 
Joseph Messinger,

Ces gestes qui manipulent, ces mots qui influencent,

2003, Paris, Éditions générales First, 414 pages.

Ce paragraphe ne vise pas à présenter le grand ouvrage d'un «spécialiste de la symbolique gestuelle», ainsi qu'il se dénomme, mais à faire connaitre, à travers lui, un type d' «analyse» de la gestuelle qui se situe à l'opposé des recherches si rigoureuses et scrupuleuses de Geneviève Calbris. Dans ce livre, en effet, tout est interprétation.

Après une première partie qui définit ce qu'est la «manipulation» et dont l'aspect pamphlétaire n'est pas le plus inintéressant, viennent les «gestes manipulateurs» puis les «mots qui influencent». Une suite de portraits, plaisants ou ridicules, défilent de page en page, sans aucune analyse, aucune précaution, tirant, avec certitude, conclusions sur conclusions de l'intuition personnelle ou, au mieux, d'une certaine expérience de psychologue d'entreprise. C'est ainsi qu'à propos de «croisements", à partir de l'indubitable intuition que «tout croisement corporel est un code de protection du territoire mental», on apprend que «si votre interlocuteur est un homme droitier défensif (croisement du bras gauche dominant le droit) et que sa jambe gauche couvre la droite, il exprime l'échec de sa manœuvre», mais «s'il s'agit d'un homme droitier offensif (croisement du bras droit dominant le gauche) et que la jambe droite couvre la gauche, l'attitude est clairement calculatrice», que «doigts croisés, index pointés en avant et collés l'un à l'autre [est un] geste inattendu indiquant un donneur de leçon capable de couper les cheveux en quatre» et encore que «le croisement des doigts en chevron est un signal de trac pur et dur». Chapitre index, vous apprendrez que «tout contact entre les lèvres et l'un des index provoque une coupure de l'intelligence logique et de la réflexion qui l'accompagne» et vous aurez droit à : «l'index amoureux», «l'index barbichette», «l'index bluffeur», «l'index faux témoin», «l'index fleuret» etc. Psychologie intuitive des attitudes et non pas étude des "gestes qui manipulent», cet ouvrage non scientifique est, au bout du compte, simplement marrant. L'analyste en gestuelle peut se dispenser de le lire.

Maurice Tournier

Gilbert Rist (dir.),

Les mots du pouvoir, sens et non-sens de la rhétorique internationale, 2002, Nouveaux Cahiers de l'IUED, 208 pages.

Dans ce numéro des Nouveaux Cahiers de l'IUED, les auteurs, venus de divers horizons disciplinaires (sociologie politique, relations internationales, 
économie, linguistique), proposent une analyse critique et engagée des productions discursives que les principales institutions du système onusien consacrent aux enjeux actuels du développement. On y trouvera les contributions de Gilbert Rist, Marie-Dominique Perrot, Francine Mestrum, Pierre Englebert, Frédéric Lapeyre, Dominique Maingueneau, Pierre de Sernaclens, Jean-Luc Alber, Christian Comeliau, Antoine Maurice, Béatrice Steiner et enfin Branislav Gosovic dans un entretien accordé à Delphine Bordier. Ces différents points de vue convergent vers un même constat, au moins quant à la réalité contemporaine d'un discours international marqué par de couteuses stratégies d'effacement et de neutralisation dans lesquelles se reflètent des enjeux politiques et idéologiques fondamentaux.

Plusieurs analyses mettent tout d'abord en évidence ce qui semble désormais caractériser une «rhétorique internationale» en perte de référents et en proie à la double logique du flou et de la rigidité. Ainsi, la répétitivité sémantique, la prévisibilité et la stéréotypisation, le manichéisme idéologique, sont autant de mécanismes discursifs qui enferment les textes dans un «style formulaire» ne laissant guère de place au débat ou au changement. Ce profil rhétorique pourrait certes être interprété comme une conséquence directe de l'universalité proclamée d'un discours qui se situe de fait en dehors de l'Histoire, c'est-à-dire en dehors des histoires singulières et parfois antagonistes, ainsi que de leurs interactions ; un discours devenu aveugle à la diversité humaine et sociale, sourd aux inégalités criantes qui divisent le monde. «L'universalité est ainsi instaurée aux dépens des réalités historiques concrètes» (Gilbert Rist, p. 13). Ce qui se joue pourtant dans l'énonciation de cette universalité ignorant par nécessité toute forme de division, et dont l'existence ne peut être que théorique, c'est bien la neutralisation et l'euphémisation des réalités socio-économiques et historiques dont la politique mondiale ne peut se dispenser dans le règlement des problèmes posés par le développement.

Mais certains auteurs voient aussi dans les mécanismes discursifs qui structurent la rhétorique internationale l'émergence d'une «langue» singulière, héritière d'une longue tradition diplomatique et dont la vocation, contrairement à celle de la désormais célèbre «langue de bois», violente et idéologiquement rigide, serait de n'engager à rien au point d'appeler toutes sortes d'interprétations - fussent-elles contradictoires. Cette langue, dite parfois «de coton», n'en est pas moins chargée d'idéologie, car derrière l'apparente neutralité des nombreuses nominalisations qui permettent à la magie du verbe d'opérer en objectivant les actions et les choix politiques, derrière l'apparente évidence des concepts figés qui prolifèrent («capital social», «lutte contre la pauvreté»...) et des grandes catégorisations, c'est une conception subjective du monde que l'on impose, au profit d'une orientation néo-libérale du développement et des 
pratiques politiques qu'elle implique, lesquelles devraient pouvoir rester discutables.

Que l'on ne s'y trompe pas, nous disent les auteurs: à force de douceur, le «coton» pourrait devenir un facteur d'enfermement de la pensée. La naturalisation des idées a en effet un cout: il rend la contradiction impossible pour un destinataire sur lequel le discours trouve ici un moyen singulier d'exercer une forme de contrôle social.

Au-delà du constat et pour comprendre ces différents mécanismes, d'autres auteurs rappellent la nécessité de s'intéresser aux contraintes politiques et institutionnelles qui entourent et déterminent la production du discours international. La rhétorique du système onusien résulte en effet du consensus, une pratique politique qui constitue encore aujourd'hui le socle de la société mondiale. Elle témoigne donc de la difficulté à réunir dans un même sens commun une multitude d'acteurs engagés dans des relations de coopération et surtout de concurrence. Or c'est aux institutions internationales à vocation universelle qu'incombe la tâche de convertir cette multitude hétérogène en ce qui n'est, au final, qu'une fiction: l'unité indivisible de la «communauté internationale». Parce que sur lui repose la cohérence même de cette «communauté» improbable, le discours fonctionne dans une forme de dénégation du conflit, par conséquent sur le mode de la globalisation, de l'approximation, en somme sur le mode d'une prudence souvent dénoncée mais dont dépendent la légitimité et l'existence des instances qui l'énoncent. Car la «rhétorique internationale» est un outil d'auto-légitimation, une compétence qui autorise les locuteurs institutionnels onusiens à prendre la parole au sein de l'espace public. Skeptron symbolique, elle est aussi le signe de l'autorité et du pouvoir de ceux qui l'énoncent.

Mais les institutions internationales à vocation universelle ont-elles un pouvoir et une autorité, leur discours a-t-il vocation à fonder un ordre social à l'échelle du monde? Si la rhétorique internationale, fondamentalement politique, est vouée à légitimer une position institutionnelle et des représentations particulières du monde et, si elle exerce par conséquent un pouvoir symbolique sur les membres de la communauté à laquelle elle s'adresse, elle reste avant tout l'objet d'intenses jeux de négociations et de marchandages qui se résolvent souvent au profit des positions dominantes qu'occupent certains acteurs, les ÉtatsUnis notamment. Ainsi l'autorité de ce discours, en dépit de sa prétention à l'universalité, ne peut être que partielle, voire factice. De ce fait, comme le souligne Dominique Maingueneau, à qui l'on doit le concept de «discours constituant», la prétention à se situer au-delà du politique et le désir d'universalité ne peuvent suffire à la rhétorique internationale pour fonder un ordre social, jouer un rôle constituant dans la société mondiale. Parce qu'il est issu du consensus et non pas de la recherche commune d'un absolu, parce qu'en réalité il reste 
destiné aux experts et non au destinataire universel, le discours des institutions internationales à vocation universelle ne peut être qu'un simulacre de discours constituant et feindre l'autorité des discours fondateurs.

Reste qu'à cette non-puissance les instances internationales ne peuvent substituer que les mots: une rhétorique qui constitue à la fois l'essentiel de leur pouvoir et la garantie de sa préservation.

\section{Fabienne Pierre}

Pascal Marchand,

L'analyse du discours assistée par ordinateur, 1998, Paris, Armand Colin, 222 pages.

Le titre de cet ouvrage se veut résolument généraliste. Pascal Marchand, du Groupe de Recherche sur la Parole de l'Université Paris 8-Saint-Denis, propose une recension des applications de l'outil informatique à l'analyse du discours et, plus largement, à l'ensemble des sciences humaines et sociales qui s'intéressent à l'étude de données verbales. Le volume présente d'abord les «concepts, méthodes et outils», qui sont illustrés, au travers d'un exemple d'application, dans une seconde partie.

Les cinq premiers chapitres sont autant d'axes pour l'analyse discursive auxquels l'outil informatique peut apporter des éléments de réponse. La part belle est faite à la statistique lexicale, le chapitre qui s'y rapporte représentant près de la moitié de cet ensemble. Le premier chapitre présente succinctement ce que l'auteur nomme les «analyses para-verbales». Il y aborde certains aspects de l'analyse conversationnelle, en termes de verbalisation, de successions de tours de parole et de comportements verbaux, puis définit quelques problématiques relevant de la phonologie, citant des travaux menés sur l'intonation ou des recherches en psychologie sociale et en sociolinguistique, par exemple celles de Labov, en particulier son analyse de la prononciation dans les différentes couches socioprofessionnelles new-yorkaises. Le deuxième chapitre, sur les analyses lexicales, s'appuie sur des travaux nombreux et divers, qui fournissent des repères conceptuels, abondamment nourris de citations de Muller, Benveniste, Bronckart, Bally, Saussure, Harris, etc. Il s'inspire largement du manuel bien connu de Ludovic Lebart et André Salem ${ }^{12}$, revient sur les différentes approches du lexique (psychologique, pragmatique, linguistique) puis définit les notions élémentaires d'unité lexicale, d'index, de forme graphique, avant d'aborder des aspects tels que la richesse du vocabulaire, la connexion et la distance lexicale, les concordances,

12. L. Lebart et A. Salem, 1994, Statistique textuelle, Paris, Dunod. 